\title{
the Application of tioz/zno nanocomposite as a New Trapping Media for Solid Phase Extraction of $u$ and th
}

\author{
Funda AYDIN ${ }^{1, *}$, Erkan YILMAZ ${ }^{2,3}$, Gülay DEMIRKIRAN ${ }^{1}$, Zeliha ERBAŞ ${ }^{4}$, \\ Mustafa SOYLAK ${ }^{4}$ \\ ${ }^{1}$ Van Yüzüncü Y1l University, Faculty of Pharmacy, Department of Basic Sciences, 65080, Van, \\ Turkey \\ ${ }^{2}$ Erciyes University, Faculty of Pharmacy, Department of Basic Sciences, Kayseri, Turkey \\ ${ }^{3}$ Erciyes University, Nanotechnology Research Center, Kayseri, Turkey \\ ${ }^{4}$ Erciyes University, Faculty of Sciences, Department of Chemistry, 38039, Kayseri, Turkey \\ * faydin@yyu.edu.tr
}

\begin{abstract}
Selecting of an appropriate sorbent for target trace analyte/analytes is an important factor to obtain high selectivity and quantitative recovery results in the solid phase extraction (SPE) studies. In recent years, nanometer-sized metal oxide materials which have high surface area and active sites for interactions with analyte/analytes have gained the attention of scientists due to their excellent sorption capacity in SPE applications. Metal oxide nanoparticles (NPs) such as $\mathrm{TiO}_{2}, \mathrm{Al}_{2} \mathrm{O}_{3}, \mathrm{ZrO}_{2}, \mathrm{CeO}_{2}, \mathrm{MnO}, \mathrm{CuO}, \mathrm{SiO}_{2}$..etc have been studied as solid-phase materials in preconcentration and separation of trace organic or inorganic species in batch and column modes.
\end{abstract}

In this study, we aimed to develop a new SPE batch method based on using $\mathrm{TiO}_{2} / \mathrm{ZnO}$ nanocomposite for determination of uranium and thorium radionüclides in water and rock samples by ICP-MS. Firstly, the nanosorbent was synthesized via a hydrothermal technique and characterization of the new material was carried out using Raman, XRD, SEM-EDX and SEM-mapping analyses. All experimental variables affecting recoveries including $\mathrm{pH}$, amount of nanosorbent, volume of chelating agent, type and volume of eluting solvent, durations of vortex, ultrasound bath and cenrifugation, sample volume and interference effect were optimized using model solutions. The accuracy of the new method was checked using certified reference materials (CRMs). Addition/recovery tests were also applied in optimum condition to determine both the applicability of the developed method and the contents of radionuclides in real water and geological rock samples by ICP-MS and satisfactory results were obtained from the experimental studies.

Keywords: Nanometarial, SPE, radionuclides, separation/preconcentration, ICP-MS 